\title{
Docetaxel with or without Ramucirumab after Platinum-Based Chemotherapy and Checkpoint Inhibitors in Advanced Urothelial Carcinoma: A Pre-Specified Subgroup Analysis from the Phase 3 RANGE Trial
}

Alexandra Drakakia,*, Conor J. Kirby ${ }^{\mathrm{b}, 1}$, Michiel S. van der Heijden ${ }^{\mathrm{c}}$, Daniel P. Petrylak ${ }^{\mathrm{d}}$, Thomas Powles $^{\mathrm{e}}$, Kim N. Chi ${ }^{\mathrm{f}}$, Aude Fléchong ${ }^{\mathrm{g}}$, Andrea Necchi ${ }^{\mathrm{h}}$, Lajos Géczi ${ }^{\mathrm{i}}$, Jae-Lyun Leej, Georgios Gakis $^{\mathrm{k}}$, Sergio Bracarda ${ }^{\mathrm{l}}$, Simon Chowdhury ${ }^{\mathrm{m}}$, Chia-Chi Lin ${ }^{\mathrm{n}}$, Daniel Keizman ${ }^{\circ}$, Ulka N. Vaishampayan ${ }^{\mathrm{p}}$, Annamaria H. Zimmermann ${ }^{\mathrm{b}}$, Katherine Bell-McGuinn ${ }^{\mathrm{b}}$ and Daniel Castellano ${ }^{\mathrm{q}}$

${ }^{\mathrm{a}}$ UCLA Medical Center, Los Angeles, CA, USA

${ }^{\mathrm{b}}$ Eli Lilly and Company, Indianapolis, IN, USA

${ }^{\mathrm{c}}$ Netherlands Cancer Institute-Antoni van Leeuwenhoek Hospital, Amsterdam, The Netherlands

${ }^{\mathrm{d}}$ Yale University School of Medicine, New Haven, CT, USA

${ }^{\mathrm{e}}$ Barts Cancer Institute, Queen Mary University of London, London, UK

${ }^{\mathrm{f}}$ British Columbia Cancer Agency, Vancouver, British Columbia, Canada

${ }^{\mathrm{g}}$ Centre Léon Bérard, Lyon, France

${ }^{\mathrm{h}}$ Fondazione IRCCS Istituto Nazionale dei Tumori, Milan, Italy

${ }^{\mathrm{i}}$ National Institute of Oncology, Budapest, Hungary

${ }^{\mathrm{j}}$ Asan Medical Centre, University of Ulsan College of Medicine, Seoul, Korea

${ }^{\mathrm{k}}$ University Hospital Würzburg, Würzburg, Germany

${ }^{1}$ Azienda Ospedaliera S. Maria, Terni, Italy

${ }^{\mathrm{m}}$ Sarah Canon Research Institute UK Ltd, London, UK

${ }^{\mathrm{n}}$ National Taiwan University Hospital, Taipei, Taiwan

${ }^{\circ}$ Meir Medical Center, Kfar Saba, Affiliated with the Sackler School of Medicine, Tel Aviv University,

Tel Aviv, Israel

${ }^{\mathrm{p}}$ Karmanos Cancer Institute, Detroit, MI, USA

${ }^{\mathrm{q}}$ Hospital Universitario 12 de Octubre, Madrid, Spain

Received 30 September 2019

Accepted 25 November 2019

\footnotetext{
${ }^{1}$ Present address: Marian University College of Osteopathic Medicine.

*Correspondence to: Alexandra Drakaki, MD, PhD, Assistant Professor of Medicine and Urology, Director - Genitourinary Medical Oncology Program, Co-Director of Research, GU Trials
}

Program, Division of Hematology/Oncology and Institute of Urologic Oncology, UCLA, Wasserman Building, 300 Stein Plaza, Los Angeles, 90095 CA, USA. Tel.:+1 310829 5471; Fax: 310 829 6192; E-mail: ADrakaki@mednet.ucla.edu. 


\begin{abstract}
.
BACKGROUND: The phase 3 RANGE trial found ramucirumab/docetaxel improved progression-free survival (PFS) versus placebo/docetaxel (median 4.1 vs 2.8 months; hazard ratio $[\mathrm{HR}]=0.757, p=0.0118$ ) for treatment of platinum-refractory metastatic urothelial carcinoma (UC). Some patients received an immune checkpoint inhibitor (ICI) prior to RANGE. In other studies, unselected patients with platinum-refractory UC exhibited an overall response rate (ORR) of $15-31 \%$ to ICIs. OBJECTIVE: Efficacy and safety data from the subgroup of patients treated with prior ICI were examined using prespecified analyses to compare outcomes between RANGE treatment arms.

METHODS: Randomized, double-blind RANGE study $(\mathrm{n}=530)$ took place July 2015-April 2017 in 23 countries. Fortyfive patients $(8.5 \%)$ received prior ICI. PFS was evaluated using the Kaplan-Meier method and unstratified Cox proportional hazards model.

RESULTS: 17 ramucirumab/docetaxel arm, 28 placebo/docetaxel arm patients were treated with an ICI. The prior-ICI ramucirumab subgroup had worse Bellmunt scores at baseline versus placebo (score of 2-3:70.6\% vs 25\%, respectively). Most patients (84.4\%) received the ICI immediately following platinum and immediately prior to RANGE. ORR to prior ICI was 6.7\% Responses were achieved by $5 / 17$ (29.4\%) on ramucirumab/docetaxel, compared to $2 / 28$ (7.1\%) on placebo/docetaxel. Median PFS was 3.15 months on ramucirumab/docetaxel versus 2.73 months on placebo/docetaxel $(\mathrm{HR}=0.786,95 \%$ $\mathrm{CI}=0.404-1.528, p=0.4877$ ). The frequency of grade $\geq 3$ adverse events was similar between arms. Limitations include sample size and treatment setting of the analyzed population.
\end{abstract}

CONCLUSIONS: Ramucirumab/docetaxel may provide a clinical benefit with acceptable safety in the third-line setting for metastatic UC patients whose disease has progressed on both prior platinum chemotherapy and ICI therapy.

Keywords: Immune checkpoint inhibitor, platinum-refractory, ramucirumab, urothelial carcinoma, VEGFR inhibitor

\section{INTRODUCTION}

Platinum-based chemotherapy remains the standard of care for patients with metastatic urothelial carcinoma (UC) and good performance status; however, most patients become platinum-refractory and their subsequent management remains a challenge. Prognosis is poor for these patients, with overall response rates (ORRs) $<20 \%$ and overall survival (OS) ranging from 6-9 months with taxane or vinflunine single-agent chemotherapy [1-3]. Five immune checkpoint inhibitors (ICIs) targeting the programmed death-ligand 1/programmed death-1 (PD-L1/PD-1) axis have been approved in platinum-refractory UC. Of these, only pembrolizumab has shown a significant OS benefit compared to chemotherapy in a randomized phase 3 trial [4]. The remaining four ICIs were approved based upon phase $1 / 2$ or phase 2 response and duration of response data with 15-31\% ORRs in unselected patients [5-8]. Unfortunately, many of these cases do not respond to PD-L1/PD-1-directed therapy, thus there is a significant unmet medical need for patients progressing following platinum and ICI therapies.

Ramucirumab is a fully human IgG1 monoclonal antibody VEGFR-2 antagonist. A combination of ramucirumab and docetaxel was compared to docetaxel and placebo in patients with platinumrefractory metastatic UC in the randomized, double-blinded, phase 3, RANGE trial. The trial met its primary progression-free survival (PFS) endpoint; the ramucirumab arm significantly prolonged PFS versus the placebo arm (median 4.1 mo [95\% confidence interval $(\mathrm{CI})=3.0-4.5]$ vs $2.8 \mathrm{mo}[2.6-3.0]$; hazard ratio $[\mathrm{HR}]=0.757,95 \% \mathrm{CI}=0.607-0.943$; $p=0.0118$ ). OS was not significantly improved but did show a positive trend in favor of ramucirumab (median 9.4 mo [95\% CI $=7.9-11.4]$ vs 7.9 mo $[95 \% \mathrm{CI}=7.0-9.3]$; stratified $\mathrm{HR}=0.887$ [95\% $\mathrm{CI}=0.724-1.086], p=0.2461$ ) [9]. Due to the statistical gated design, the ORR was not formally tested but showed a numerical improvement in the ramucirumab arm with non-overlapping CIs $(24.5 \%, 95 \%$ $\mathrm{CI}=18.8-30.3$ vs $14.0 \%, 9.4-18.6$ ).

Of interest, patients who had received prior platinum and ICI inhibitor therapy were eligible for RANGE. Due to limited availability of immune therapy at the time of enrollment (July 2015 through April 2017), this subgroup represented only 45 of the 530 patients. Pre-specified subgroup analyses are presented herein.

\section{PATIENTS AND METHODS}

\section{Study design and procedures}

The design of the RANGE phase 3 trial (ClinicalTrials.gov, NCT02426125) has been reported previously [10]. In brief, patients with advanced 
or metastatic UC who progressed during or after platinum-based chemotherapy were enrolled. Previous treatment with one ICI was permitted. Patients who had received an ICI were permitted to enroll if they were $\leq 24$ months from the end of a platinumcontaining regimen compared to $\leq 14$ months from the end of a platinum-containing regimen if they had not received an ICI. Patients were ineligible if they had received more than one prior systemic chemotherapy in the relapsed or metastatic setting. Patients were randomized $(1: 1)$ to receive docetaxel $\left(75 \mathrm{mg} / \mathrm{m}^{2}\right)\left(60 \mathrm{mg} / \mathrm{m}^{2}\right.$ in Korea, Taiwan, and Japan) and growth factor support with ramucirumab $(10 \mathrm{mg} / \mathrm{kg})$ or placebo on day 1 of a $21-\mathrm{d}$ cycle until disease progression or other discontinuation criteria. The primary endpoint was investigator-assessed PFS. Secondary endpoints included OS, overall response rate (RECIST v1.1) [11], and safety (NCI CTCAE v 4.0) [12]. Radiographic assessment occurred every 6 weeks. The trial conformed with the Declaration of Helsinki and the International Conference on Harmonisation Guidelines for Good Clinical Practice. The ethics committee of all participating trial centers approved the protocol. All patients provided written informed consent.

\section{Statistical analyses}

OS and PFS were evaluated by treatment arm for the prior ICI patients using the Kaplan-Meier method [13]. The unstratified Cox proportional hazards model was used to estimate HR and $95 \%$ CI [14]. ORRs and adverse event rates were reported descriptively.

\section{RESULTS}

\section{Baseline characteristics}

Forty-five of the 530 patients $(8.5 \%)$ in the intent-to-treat (ITT) population received a prior ICI, 17 patients on the ramucirumab/docetaxel arm and 28 patients on the placebo/docetaxel arm, comprising the intent-to-treat, prior-ICI population (Fig. S1, Supplementary Information). Of this patient population, 16 ramucirumab/docetaxel arm patients and 27 placebo/docetaxel arm patients were treated and comprise the safety prior-ICI population (Fig. S1, Supplementary Information). The majority were male $(77.8 \%)$, had a median age of 66 years, were predominately white $(88.9 \%)$, and were from Europe/Other $(71.1 \%)$ versus North
Table 1

Baseline characteristics among patients with prior ICI by RANGE treatment subgroups

\begin{tabular}{|c|c|c|}
\hline & $\begin{array}{c}\text { Ramucirumab+ } \\
\text { docetaxel } \\
(n=17)\end{array}$ & $\begin{array}{c}\text { Placebo+ } \\
\text { docetaxel } \\
(n=28)\end{array}$ \\
\hline Median age, yr (range) & $66(34-85)$ & $65(47-77)$ \\
\hline Male, n (\%) & $12(70.6)$ & $23(82.1)$ \\
\hline \multicolumn{3}{|l|}{ Race, n (\%) } \\
\hline White & $16(94.1)$ & $24(85.7)$ \\
\hline Asian & $1(5.9)$ & $4(14.3)$ \\
\hline \multicolumn{3}{|l|}{ ECOG performance status, $\mathrm{n}(\%)$} \\
\hline 0 & $1(5.9)$ & $12(42.9)$ \\
\hline 1 & $16(94.1)$ & $16(57.1)$ \\
\hline \multicolumn{3}{|l|}{ Geography, n (\%) } \\
\hline Europe & $14(82.4)$ & $18(64.3)$ \\
\hline North America & $2(11.8)$ & $6(21.4)$ \\
\hline East Asia & $1(5.9)$ & $4(14.3)$ \\
\hline \multicolumn{3}{|l|}{ Primary tumor site, $\mathrm{n}(\%)$} \\
\hline Bladder & $13(76.5)$ & $17(60.7)$ \\
\hline Renal pelvis & $2(11.8)$ & $4(14.3)$ \\
\hline Ureter & $2(11.8)$ & $5(17.9)$ \\
\hline Other & 0 & $2(7.1)$ \\
\hline \multicolumn{3}{|l|}{ Duration of disease (months) ${ }^{\mathrm{a}}$} \\
\hline Median & 24.1 & 17.1 \\
\hline Interquartile range (Q3-Q1) & $30.8-19.5$ & $37.8-12.7$ \\
\hline \multicolumn{3}{|l|}{ Number of metastatic sites, n (\%) } \\
\hline 1 & $2(11.8)$ & $6(21.4)$ \\
\hline 2 & $6(35.3)$ & $11(39.3)$ \\
\hline 3 & $3(17.6)$ & $8(28.6)$ \\
\hline 4 & $4(23.5)$ & 0 \\
\hline 5 & $2(11.8)$ & $1(3.6)$ \\
\hline Missing & 0 & $2(7.1)$ \\
\hline \multicolumn{3}{|l|}{ Visceral metastasis, $\mathrm{n}(\%)$} \\
\hline Yes & $14(82.4)$ & $20(71.4)$ \\
\hline Liver & $10(58.8)$ & $7(25.0)$ \\
\hline Lung & $8(47.1)$ & $15(53.6)$ \\
\hline Bone & $5(29.4)$ & $5(17.9)$ \\
\hline Kidney & 0 & $2(7.1)$ \\
\hline Adrenal gland & 0 & $1(3.6)$ \\
\hline Spleen & $1(5.9)$ & $1(3.6)$ \\
\hline Other & $4(23.5)$ & $3(10.7)$ \\
\hline No & $3(17.6)$ & $8(28.6)$ \\
\hline Lymph node only & $2(11.8)$ & $3(10.7)$ \\
\hline \multicolumn{3}{|l|}{ Bellmunt risk factors ${ }^{\mathrm{b}}, \mathrm{n}(\%)$} \\
\hline 0 & $1(5.9)$ & $10(35.7)$ \\
\hline 1 & $4(23.5)$ & $11(39.3)$ \\
\hline 2 & $12(70.6)$ & $6(21.4)$ \\
\hline 3 & 0 & $1(3.6)$ \\
\hline
\end{tabular}

${ }^{a}$ Defined as months from first diagnosis of cancer to randomization. ${ }^{b}$ Bellmunt risk factors include an Eastern Cooperative Oncology Group (ECOG) performance status $>0$, presence of liver metastases, and haemoglobin concentration $<10 \mathrm{~g} / \mathrm{dL}$.

America (17.8\%) or East Asia (11.1\%) (Table 1). There was a higher percentage of patients on the ramucirumab/docetaxel arm with a poorer prognosis compared to the placebo/docetaxel arm: baseline Bellmunt risk factors score [15] of 2 or 3 was $70.6 \%$ compared to $25 \%$, baseline Eastern Cooperative Oncology Group performance status (ECOG PS) of 1 was $94.1 \%$ versus $57.1 \%$, liver metastases were present in $58.8 \%$ compared to $25.0 \%$, and 3 or more metastatic sites were present in $52.9 \%$ versus $32.1 \%$ of patients (with available data). The median 
duration of disease, defined as months from first diagnosis of cancer to randomization, was longer in the ramucirumab/docetaxel versus placebo/docetaxel arms (24.1 mo, interquartile range $[\mathrm{IQR}]=19.5-30.8$ mo vs $17.1 \mathrm{mo}, \mathrm{IQR}=12.7-37.8$ ).

\section{Prior ICI therapies}

Most patients (84.4\%) received the ICI immediately following platinum and immediately prior to RANGE. Most patients received atezolizumab or pembrolizumab (Table 2). Median duration of the prior ICI therapy was 3.0 months versus 3.8 months, with ORRs to prior ICI therapy of 5.9\% versus $7.1 \%$, for the ramucirumab/docetaxel arm and placebo/docetaxel arm, respectively (Table 2).

\section{Efficacy measures}

Five of the 17 (29.4\%) ramucirumab/docetaxel arm patients had a partial response (PR) as the best overall tumor response, with a $47-76 \%$ reduction in tumor size (Table 3, Fig. 1). Six additional patients $(35.3 \%)$ had stable disease (SD), for a disease control rate $(\mathrm{PR}+\mathrm{SD})$ of $64.7 \%$. Response to ramucirumab/docetaxel appeared independent of metastatic disease site (Fig. 1, lower panel). Fewer responses were seen in the placebo/docetaxel arm with partial responses in $7.1 \%$ of patients $(2 / 28$ patients); the stable disease rate was $57.1 \%(16 / 28$ patients) for a disease control rate (PR+SD) of $64.2 \%$. Duration of response was longer on the ramucirumab arm (median $4.9 \mathrm{mo}$; 95\% CI=3.9-6.7) than the placebo arm (median 3.5 mo: $95 \% \mathrm{CI}=2.8-4.2$ ) (Table 3). Tumor response in both arms was similar in upper and lower UC. Four of the 5 responders on the ramucirumab arm had a Bellmunt risk factors score of 2 (Table 3). Duration of treatment for each patient on both treatment arms is summarized in Figure S2 (Supplementary Information).

Median PFS was 3.15 months on ramucirumab/ docetaxel and 2.73 months on placebo/docetaxel $(\mathrm{HR}=0.786,95 \% \mathrm{CI}=0.404-1.528, p=0.4877)$ (Table 4; Fig. S3, Supplementary Information). At 3 and 6 months, the estimated proportion of patients who were progression free was $53.8 \%$ and $31.4 \%$ on the ramucirumab/docetaxel arm, respectively, and $31.1 \%$ and $11.7 \%$ on the placebo/docetaxel arm, respectively (Table 4). OS was 8.90 months on the ramucirumab/docetaxel arm and 8.11 months on the placebo/docetaxel arm $(\mathrm{HR}=1.227,95 \%$ $\mathrm{CI}=0.630-2.390, p=0.5445)$ (Table 4; Fig. S3, Supplementary Information).

\section{Safety}

Exposure to RANGE treatment, ramucirumab or placebo and docetaxel, was similar between treatment arms for the prior ICI subgroups (Table S1). The median duration of therapy was 10.8 weeks $(\mathrm{IQR}=6.0-25.4)$ for ramucirumab arm patients and 12.0 weeks $(\mathrm{IQR}=7.0-19.0)$ for placebo arm patients.

The frequency of any grade and grade $\geq 3$ treatment-emergent adverse events (TEAEs) was

Table 2

Summary of prior ICI by RANGE treatment subgroups

\begin{tabular}{lcc}
\hline & $\begin{array}{c}\text { Ramucirumab+ } \\
\text { docetaxel } \\
(n=17)\end{array}$ & $\begin{array}{c}\text { Placebo+ } \\
\text { docetaxel } \\
(n=28)\end{array}$ \\
\hline Prior ICI, n (\%) & $10(58.8)$ & $11(39.3)$ \\
Atezolizumab (anti-PD-L1) & $5(29.4)$ & $10(35.7)$ \\
Pembrolizumab (anti-PD-1) & $1(5.9)$ & $1(3.6)$ \\
BGBA317 (anti-PD-1) & $1(5.9)$ & $2(7.1)$ \\
Durvalumab (anti-PD-L1) & 0 & $1(3.6)$ \\
Durvalumab and tremelimumab (anti- CTLA-4) & 0 & $3(10.7)$ \\
Nivolumab (anti-PD-1) & $3.0(1.5-5.5)$ & $3.8(2.8-5.7)$ \\
Median duration of prior ICI, mo (IQR) & & \\
Tumor response to ICI, $\mathrm{n}(\%)$ & 0 & 0 \\
Complete Response (CR) & $1(5.9)$ & $2(7.1)$ \\
Partial Response (PR) & $4(23.5)$ & $8(28.6)$ \\
Stable Disease (SD) & $12(70.6)$ & $18(64.3)$ \\
Progressive Disease & $1(5.9)$ & $2(7.1)$ \\
Overall response, $\mathrm{n}(\%)$ & $5(29.4)$ & $10(35.7)$ \\
Disease control (CR/PR/SD), $\mathrm{n}(\%)$ & &
\end{tabular}

Abbreviations: $\mathrm{ICI}=$ immune checkpoint inhibitor; $\mathrm{IQR}=$ interquartile range; $\mathrm{mo}=$ month; PD-1 = programmed death-1; PD-L1 = programmed death-ligand 1 . 
Table 3

Tumor response to RANGE treatments of prior ICI patients

\begin{tabular}{lcc}
\hline & $\begin{array}{c}\text { Ramucirumab+ } \\
\text { docetaxel } \\
(n=17)^{a}\end{array}$ & $\begin{array}{c}\text { Placebo+ } \\
\text { docetaxel } \\
(n=28)^{a}\end{array}$ \\
\hline $\begin{array}{l}\text { Overall tumor response, } \mathbf{n}(\%) \\
\quad \text { Complete response }\end{array}$ & 0 & $0^{\mathrm{b}}$ \\
$\quad$ Partial response & $5(29.4)$ & $2(7.1)$ \\
$\quad$ Stable disease & $6(35.3)$ & $16(57.1)$ \\
$\quad$ Progressive disease & $3(17.6)$ & $7(25.0)$ \\
$\quad$ Non-evaluable & $3(17.6)$ & $3(10.7)$ \\
Overall response rate, $\mathrm{n}(\%)$ & $5(29.4)$ & $2(7.1)$ \\
$\quad$ Disease control rate, $\mathrm{n}(\%)$ & $11(64.7)$ & $18(64.3)$ \\
Duration of response & & \\
Median, $95 \%$ CI (mo) & $4.9(3.9-6.7)$ & $3.5(2.8-4.2)$ \\
Patients with UC of the upper tract & $(n=4)$ & $(n=9)^{\mathrm{c}}$ \\
Overall response, $\mathrm{n}(\%)$ & $1(25.0)$ & $1(11.1)$ \\
Patients with UC of the lower tract & $(n=13)$ & $(n=17)^{\mathrm{c}}$ \\
Overall response, $\mathrm{n}(\%)$ & $4(30.8)$ & $1(5.9)$ \\
By number of Belmunt risk factors ${ }^{\mathrm{d}}$ & & \\
Overall response, $\mathrm{n} /$ total no. of patients with & & $1 / 10(10)$ \\
given number of Bellmunt risk factors $(\%)$ & & $1 / 11(9.1)$ \\
0 & $0 / 1(0)$ & $0 / 6(0)$ \\
1 & $1 / 4(25)$ & $0 / 1(0)$ \\
\hline
\end{tabular}

Abbreviations: $\mathrm{CI}=$ confidence interval; $\mathrm{UC}=$ urothelial carcinoma. ${ }^{\mathrm{a}}$ One of 17 patients on the ramucirumab arm and 1 among 28 on the placebo arm received no study treatment. ${ }^{b}$ At the time of PFS datalock, one placebo arm patient was categorized as complete response at cycle 6 . The next response assessment was in cycle 9 and this patient was recorded as a nontarget progressive disease. Per RECIST criteria, the overall response for this patient must therefore be a partial response rather than a complete response. ${ }^{\mathrm{c}}$ The UC of 2 patients on the placebo arm was denoted as "Other" rather than upper or lower. ${ }^{\mathrm{d}}$ The Bellmunt risk factors were Eastern Cooperative Oncology Group performance status $>0$, presence of liver metastases, and haemoglobin $<10 \mathrm{~g} / \mathrm{dl}$.
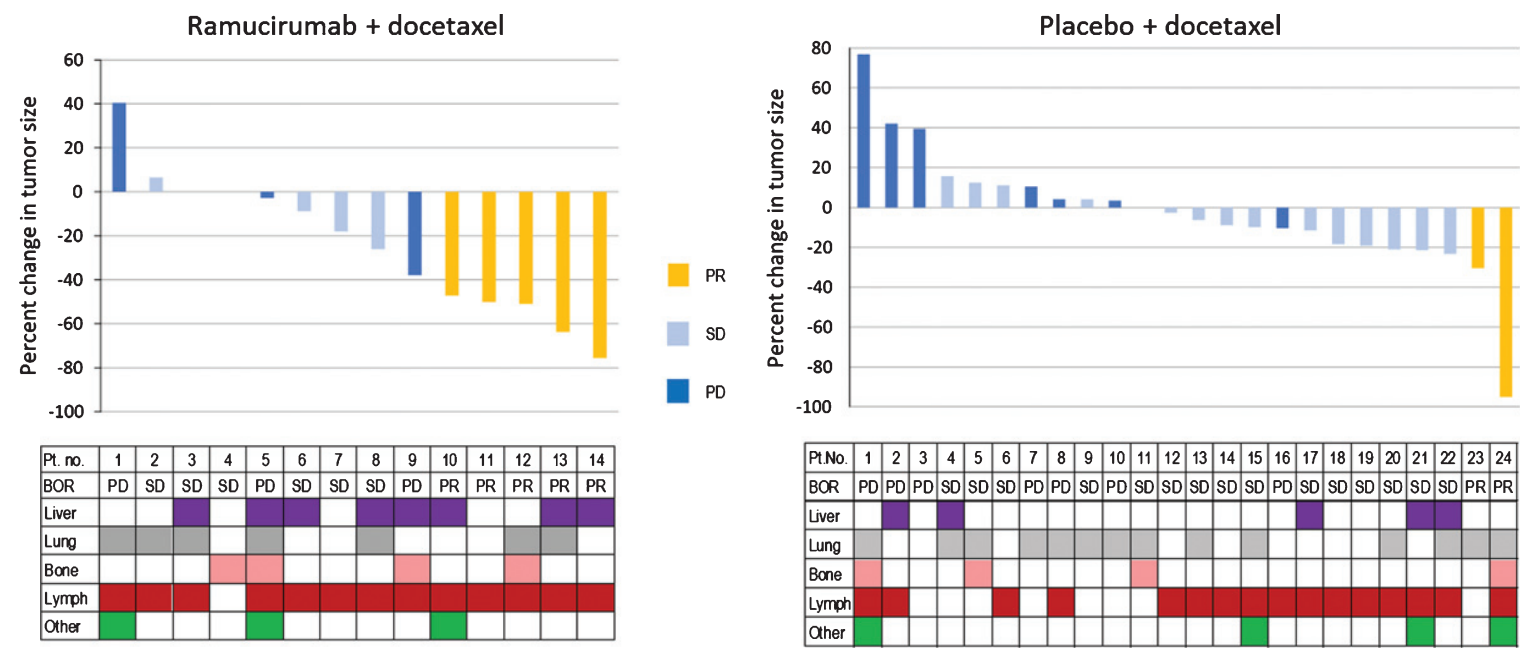

Fig. 1. Waterfall plot depicting best percent change from baseline in tumor size and best overall tumor response for prior-ICI patients by RANGE treatment arm. The prior-ICI patient population is shown by RANGE treatment arm, ramucirumab/docetaxel (left) and placebo/docetaxel (right), with the graph depicting the best relative change in tumor size (\%) and tumor response (see color key). Patients on each treatment arm were assigned an identification number; the same patient numbers are reflected in Figure S2, Supplemental Information. The chart below each Waterfall plot indicates the sites of metastases for each patient. Abbreviations: $\mathrm{PD}=$ progressive disease; $\mathrm{PR}=$ partial response; $\mathrm{SD}=$ stable disease. 
Table 4

\begin{tabular}{|c|c|c|}
\hline & $\begin{array}{c}\text { Ramucirumab }+ \\
\text { docetaxel }(n=17)\end{array}$ & $\begin{array}{c}\text { Placebo }+ \\
\text { docetaxel }(n=28)\end{array}$ \\
\hline \multicolumn{3}{|l|}{$\begin{array}{l}\text { Progression-free survival (PFS) } \\
\text { (P) }\end{array}$} \\
\hline Median, mo (95\% CI) & $3.15(1.84-6.60)$ & $2.73(1.64-2.79)$ \\
\hline$p$-value, 2 -sided, log-rank, unstratified & \multicolumn{2}{|c|}{0.4877} \\
\hline Hazard ratio (unstratified) $(95 \% \mathrm{CI})$ & \multicolumn{2}{|c|}{$0.786(0.404-1.528)$} \\
\hline 3-mo PFS rate, $\%(95 \% \mathrm{CI})$ & $53.8(26.8-74.8)$ & $31.1(14.8-48.9)$ \\
\hline 6-mo PFS rate, $\%(95 \% \mathrm{CI})$ & $31.4(10.3-55.4)$ & $11.7(3.0-27.0)$ \\
\hline \multicolumn{3}{|l|}{ Overall survival (OS) } \\
\hline Median, mo $(95 \% \mathrm{CI})$ & $8.90(2.99-11.86)$ & $8.11(4.99-12.85)$ \\
\hline$p$-value, 2-sided, log-rank, unstratified & \multicolumn{2}{|c|}{0.5445} \\
\hline Hazard ratio (unstratified) $(95 \% \mathrm{CI})$ & \multicolumn{2}{|c|}{$1.227(0.630-2.390)$} \\
\hline 3-mo OS rate, $\%(95 \% \mathrm{CI})$ & $75.0(46.3-89.8)$ & $81.2(60.5-91.7)$ \\
\hline 6-mo OS rate, $\%(95 \% \mathrm{CI})$ & $62.5(34.9-81.1)$ & $64.9(43.3-80.0)$ \\
\hline 9-mo OS rate, $\%(95 \% \mathrm{CI})$ & $50.0(24.5-71.0)$ & $44.6(25.0-62.5)$ \\
\hline
\end{tabular}

Abbreviation: $\mathrm{CI}=$ confidence interval; $\mathrm{mo}=$ month.

similar between arms in the prior-ICI safety population (Table 5; Table S2, Supplementary Information). Grade 3-5 neutropenia, diarrhea, and mucosal inflammation were only seen in the ramucirumab arm, albeit with low patient numbers: 2, 3, and 2, respectively. The incidence of grade 3-4 adverse events of special interest (AE-SIs) was similar on the two treatment arms: $12.5 \%$ ramucirumab and $11.1 \%$ placebo. However, some low-grade AE-SIs including epistaxis at $25.0 \%$ versus $3.7 \%$ and proteinuria at $18.8 \%$ versus $0 \%$ occurred more frequently on the ramucirumab arm versus the placebo arm, respectively (Table 5). There were no deaths on treatment or within 30 days of treatment discontinuation that were considered related to study treatment (Table S2, Supplementary Information).

\section{DISCUSSION}

This pre-planned RANGE subgroup analysis assessed the impact on outcomes and safety of ramucirumab added to docetaxel after disease progression on both platinum and ICI therapy. For most of these patients $(84.4 \%)$, the ICI therapy was administered after progression on platinum, thus the ramucirumab/placebo plus docetaxel treatment was a third-line treatment regimen. Third-line treatments for metastatic UC have not been thoroughly explored, although several trials are currently ongoing [16]. There are no completed randomized third-line phase 3 trials of metastatic UC treatments, and third-line phase 2 trials are limited by size and potential patient population selection bias. As ICI treatment for metastatic UC patients has become routine as second-line therapy, and results from frontline phase 3 trials evaluating ICI monotherapy and platinum combination approaches are expected in the near future, questions arise as to the efficacy and safety of post-ICI progression treatments. At the same time, there is an awareness that as disease progresses, there is an increase in tumor burden and usually a decline in performance status. Patients treated in the third-line setting tend to be frail, more vulnerable to drug-related side effects, and have disease that is increasingly refractory to additional therapies.

Among the 45 patients who received immunotherapy prior to their participation in the RANGE trial, the 17 patients randomized to the ramucirumab/docetaxel arm exhibited a numerically higher ORR than the 28 patients randomized to the placebo/docetaxel arm. Additionally, the ramucirumab-treated subgroup had a longer duration of response than the placebo group. Likewise, PFS and the 3- and 6-month PFS rates directionally favored the ramucirumab-treated arm, mirroring the results in the full population. OS was similar between treatment arms and similar to the results for the full RANGE population. This indication of a ramucirumab benefit occurred despite the ramucirumab arm having a higher percentage of patients with poorer prognosis (assessed by Bellmunt risk factors, metastatic burden, and presence of liver metastases) and lower response rate to their prior ICI therapy (6-7\% response compared to the $13-31 \%$ response observed in phase 2 and 3 trials with ICI therapies) $[4-6,8,17,18]$. While the small sample size limits measurement of statistical differences, the ramucirumab arm showed the same directional 
Table 5

Treatment-emergent adverse events and adverse events of special interest of prior-ICI patient subgroups in response to RANGE treatments ${ }^{\mathrm{a}}$

\begin{tabular}{|c|c|c|c|c|}
\hline \multirow[b]{2}{*}{$\begin{array}{l}\text { Treatment-emergent adverse } \\
\text { events (TEAEs), } n(\%)\end{array}$} & \multicolumn{2}{|c|}{$\begin{array}{c}\text { Ramucirumab+docetaxel } \\
(n=16)\end{array}$} & \multicolumn{2}{|c|}{$\begin{array}{c}\text { Placebo+docetaxel } \\
(n=27)\end{array}$} \\
\hline & Any Grade & Grade $3 / 4 / 5$ & Any Grade & Grade $3 / 4 / 5$ \\
\hline$\geq 1 \mathrm{TEAE}$ & $16(100)$ & $11(68.8)$ & $27(100)$ & $21(77.8)$ \\
\hline \multicolumn{5}{|l|}{ Blood and lymphatic disorders } \\
\hline Anemia & $4(25.0)$ & $1(6.3)$ & $8(29.6)$ & $3(11.1)$ \\
\hline Febrile neutropenia & $2(12.5)$ & $2(12.5)$ & $4(14.8)$ & $4(14.8)$ \\
\hline Neutropenia & $2(12.5)$ & $2(12.5)$ & $1(3.7)$ & 0 \\
\hline \multicolumn{5}{|l|}{ Gastrointestinal disorders } \\
\hline Diarrhea & $9(56.3)$ & $3(18.8)$ & $5(18.5)$ & 0 \\
\hline Nausea & $6(37.5)$ & 0 & $5(18.5)$ & 0 \\
\hline Stomatitis & $5(31.3)$ & 0 & $6(22.2)$ & 0 \\
\hline Constipation & $3(18.8)$ & 0 & $6(22.2)$ & 0 \\
\hline Vomiting & $3(18.8)$ & 0 & $3(11.1)$ & 0 \\
\hline Abdominal pain & $2(12.5)$ & 0 & $1(3.7)$ & 0 \\
\hline Dysphagia & $2(12.5)$ & 0 & $1(3.7)$ & 0 \\
\hline \multicolumn{5}{|l|}{ General disorders } \\
\hline Fatigue & $5(31.3)$ & 0 & $16(59.3)$ & $1(3.7)$ \\
\hline Edema peripheral & $4(25.0)$ & 0 & $2(7.4)$ & 0 \\
\hline Asthenia & $3(18.8)$ & 0 & $4(14.8)$ & 0 \\
\hline Mucosal inflammation & $3(18.8)$ & $2(12.5)$ & 0 & 0 \\
\hline Pyrexia & $3(18.8)$ & 0 & $6(22.2)$ & 0 \\
\hline Malaise & $2(12.5)$ & 0 & 0 & 0 \\
\hline \multicolumn{5}{|l|}{ Infections } \\
\hline Urinary tract infection & $6(37.5)$ & $2(12.5)$ & $6(22.2)$ & $3(11.1)$ \\
\hline Oral candidiasis & $3(18.8)$ & 0 & $1(3.7)$ & 0 \\
\hline \multicolumn{5}{|l|}{ Metabolism disorders } \\
\hline Decreased appetite & $7(43.8)$ & $1(6.3)$ & $7(25.9)$ & 0 \\
\hline \multicolumn{5}{|l|}{ Musculoskeletal/connective tissue disorders } \\
\hline Myalgia & $2(12.5)$ & 0 & $3(11.1)$ & 0 \\
\hline \multicolumn{5}{|l|}{ Nervous system disorders } \\
\hline Dysgeusia & $3(18.8)$ & 0 & 0 & 0 \\
\hline Peripheral sensory neuropathy & $2(12.5)$ & 0 & $1(3.7)$ & 0 \\
\hline \multicolumn{5}{|l|}{ Skin and subcutaneous tissue disorders } \\
\hline Alopecia & $3(18.8)$ & 0 & $7(25.9)$ & 0 \\
\hline Onycholysis & $2(12.5)$ & 0 & $2(7.4)$ & $1(3.7)$ \\
\hline Rash & $2(12.5)$ & 0 & $3(11.1)$ & 0 \\
\hline Adverse events of special interest (AE-SIs) & Any Grade & Grade 3/4/5 & Any Grade & Grade 3/4/5 \\
\hline Any AE-SI & $10(62.5)$ & $2(12.5)$ & $8(29.6)$ & $3(11.1)$ \\
\hline Bleeding/hemorrhage & $7(43.8)$ & 0 & $4(14.8)$ & $1(3.7)$ \\
\hline Epistaxis & $4(25.0)$ & 0 & $1(3.7)$ & 0 \\
\hline Gastrointestinal hemorrhage & $1(6.3)$ & 0 & 0 & 0 \\
\hline Hematuria & $1(6.3)$ & 0 & $3(11.1)$ & $1(3.7)$ \\
\hline Hemoptysis & $1(6.3)$ & 0 & 0 & 0 \\
\hline Vaginal hemorrhage $^{b}$ & $1(25.0)$ & 0 & 0 & 0 \\
\hline Hypertension & $1(6.3)$ & $1(6.3)$ & $2(7.4)$ & 0 \\
\hline Congestive heart failure & 0 & 0 & $1(3.7)$ & $1(3.7)$ \\
\hline Proteinuria & $3(18.8)$ & 0 & 0 & 0 \\
\hline Arterial thromboembolic events & 0 & 0 & $1(3.7)$ & 0 \\
\hline Venous thromboembolic evetns & $1(6.3)$ & 0 & $2(7.4)$ & $1(3.7)$ \\
\hline Renal failure & $2(12.5)$ & $1(6.3)$ & $2(7.4)$ & 0 \\
\hline
\end{tabular}

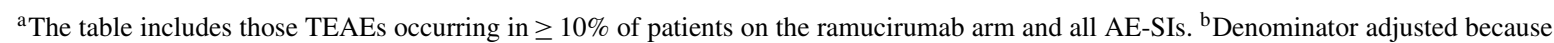
gender-specific event for females; $n=4$ for both treatment arms.

results as the full RANGE population for efficacy measures.

Safety measures assessed in the post-ICI subgroups were like those of the full RANGE population, including the incidence rate of grade 3-5 TEAEs on the ramucirumab arm (68.8\% for the post-ICI population and $65.1 \%$ for the full RANGE population). In general, those TEAEs with greater incidence among the full RANGE population (both arms) also occurred more frequently among the post-ICI subgroups. How- 
ever, the incidence of three ramucirumab arm TEAEs appeared greater in the post-ICI subgroup relative to the incidence exhibited by the ramucirumab arm of the entire population. Any grade urinary tract infection was $37.5 \%$ in the ramucirumab arm of the post-ICI subgroup versus $12.8 \%$ in the full RANGE population; grade 3/4 urinary tract infection was $12.5 \%$ in the ramucirumab arm of the post-ICI subgroup versus $4.3 \%$ in the full RANGE population (Table 5) [9]. In a similar fashion, mucosal inflammation (any grade: $18.8 \%$ vs $5.4 \%$; grade $3 / 4: 12.5 \%$ vs $1.2 \%$ ) and diarrhea (any grade: $56.3 \%$ vs $32.2 \%$; grade $3 / 4: 18.5 \%$ vs $3.5 \%$ ) were more commonly observed in the ramucirumab arm of the post-ICI subgroup than in the full RANGE population. For each of these TEAEs, the placebo arm post-ICI patients exhibited incidence like that of the full population. Of course, it must be noted that the number of patients in the ramucirumab post-ICI subgroup was low $(n=16)$ and the smaller sample size increases the magnitude of variability in incidence rates. Additional clinical data will be needed to establish if this is a real trend. Notably, a phase 1 trial of ramucirumab and pembrolizumab demonstrated no increase in toxicity over each agent individually [19], However, given the approximate 30 -d terminal half-life of checkpoint inhibitors, there exists the potential for interaction, not only from a pharmacokinetic standpoint, but also from a pharmacodynamic one as well.

The prespecified post-ICI subgroup analyses described here are limited by more than patient number. As mentioned, the treatment arms for both post-ICI subgroups were not balanced with respect to prognostic factors. In addition, the treatment arms were imbalanced in that the ramucirumab arm also had a longer median duration of disease and an imbalance in the type of prior ICI therapy, with the ramucirumab arm having a higher percentage of patients receiving atezolizumab and other anti-PD-L1 therapy. This difference may also impact the analyses as shown by a recent meta-analysis of the pivotal second-line metastatic UC trials that found evidence of efficacy and safety differences between anti-PDL1 inhibitors and anti-PD-1 inhibitors [20].

Overall, these results are of interest in that responses were achieved, and adverse events appeared manageable for this subgroup of RANGE patients. Efficacy and safety in this subgroup were consistent with the overall ITT population in RANGE [10] and support ramucirumab/docetaxel activity in the third-line setting for metastatic UC patients whose disease has progressed on both prior platinum chemotherapy and ICI therapy. Studies are ongoing evaluating the combination of platinum, gemcitabine, and ICI therapy for first-line therapy with anticipated results in the near future. If such a triplet were to become a future standard of care, the results of this subgroup in RANGE may be considered as hypothesis-generating for treatment options following a first-line chemotherapy plus ICI regimen. However, given the limited sample size and treatment setting of the analyzed population, additional prospective trials are necessary to confirm these findings.

\section{DATA SHARING STATEMENT}

Lilly provides access to all individual participant data collected during the trial, after anonymization, with the exception of pharmacokinetic or genetic data. Data are available to request 6 months after the indication studied has been approved in the US and EU and after primary publication acceptance, whichever is later. No expiration date of data requests is currently set once data are made available. Access is provided after a proposal has been approved by an independent review committee identified for this purpose and after receipt of a signed data sharing agreement. Data and documents, including the study protocol, statistical analysis plan, clinical study report, blank or annotated case report forms, will be provided in a secure data sharing environment. For details on submitting a request, see the instructions provided at www.vivli.org.

\section{ACKNOWLEDGMENTS}

The authors thank the patients, their families, and study personnel across all sites for participating in this study. They also thank Mary Dugan Wood for writing assistance. This work was supported by Eli Lilly and Company.

\section{FUNDING}

Eli Lilly and Company.

\section{AUTHOR CONTRIBUTIONS}

AD, DPP, TP, AHZ and KBM contributed to the study conception and design; AD, KNC, J-LL, UNV, AN, MvdH, TP, AF, LG, GG, SC, C-CL, DK, AHZ, $\mathrm{DC}$ and $\mathrm{SB}$ contributed to the acquisition of data; $\mathrm{AD}$, DPP, KBM, J-LL, UNV, AN, CJK, MvdH, AF, GG, 
$\mathrm{SC}, \mathrm{C}-\mathrm{CL}, \mathrm{AHZ}$ and SB contributed to the analysis and interpretation of data; AD, DPP, TP and KBM drafted the manuscript; AD, DPP, KNC, KBM, JLL, UNV, AN, CJK, MvdH, AHZ, TP, AF, LG, GG, SC, C-CL, DK, DC and SB contributed to the critical revision of the manuscript for important intellectual content; AHZ contributed to the statistical analysis.

\section{CONFLICT OF INTEREST}

AD reports reimbursement for ASCO GU presentation expenses from Eli Lilly during the conduct of the study; Kynan Pharma, Allogene, and Urogen stock or equities ownership; personal fees from AstraZeneca and BMS; and grants from Kite Pharma, all outside the submitted work.

$\mathrm{MvdH}$ reports grants to his institution and personal fees from AstraZeneca, BMS, and Roche; and personal fees from MSD and Janssen, all outside the submitted work.

DP reports grant support and consultant fees from Ada Cap, Astellas, AstraZeneca, Bayer, BMS, Clovis, Eli Lilly, Pfizer, Roche, and Seattle Genetics; grant support from Endocyte, Genetech, Innocrin, MedImmune, Merck, Novartis, Progenics, and Sanofi Aventis; consultant fees from Amgen, Boehringer Ingelheim, Exelixis, Incyte, Janssen, Pharmacyclics, and Urogen; and stock/investment with Bellicum and Tyme.

TP reports grants and honorarium from AstraZeneca and Roche; and honorarium from BMS, Ipsen, Exelixis, Merck, Pfizer, Novartis, Incyte, Seattle Genetics, and MSC, all outside the submitted work.

$\mathrm{KNC}$ reports grants from Eli Lilly during the conduct of the study; grants and personal fees from Janssen, Astellas, Essa, Sanofi, Bayer, Roche, and AstraZeneca, outside the submitted work.

AF reports personal fees from MSD, AstraZeneca, Bayer, and Janssen, outside the submitted work.

SC reports personal fees and non-financial support from Johnson \& Johnson, Astellas, Sanofi; and personal fees from Clovis, all outside the submitted work.

AN reports grants and personal fees from Merck, AstraZeneca, and Rainier Therapeutics; and personal fees from Roche, BMS Incyte, Bayer, and Clovis Oncology, all outside the submitted work.

J-LL reports personal fees from Astellas Pharma Korea, BMS Korea, Amgen Korea, Sanofi Aventis Korea, and Novartis Korea; grants and personal fees
Pfizer Korea, Roche, and AstraZeneca; non-financial support from Pfizer; and grants from MSD, all outside the submitted work.

SB reports advisory board membership for Pfizer, BMS, Norvatis, MSD, Roche, Astellas, Janssen, and Ipsen; travel accommodation with Pfizer, BMS, Roche, Astellas, Janssen, Ipsen, AstraZeneca, and Exelixis; and honoraria for talks from Astellas, Janssen and BMS.

SC reports personal fees and non-financial support from Johnson \& Johnson, Astellas, and Sanofi; and personal fees from Clovis, all outside the submitted work.

UNV reports grants and personal fees from BMS, Exelixis, and Bayer; personal fees from Sanofi and Pfizer; and grants from Astellas, all outside the submitted work.

CJK was employed by Eli Lilly during the period of his contributions to this manuscript.

$\mathrm{KBM}, \mathrm{AHZ}$ are employees and stockholders of Eli Lilly.

DC, DK, C-CL, GG, LG have nothing to disclose.

\section{SUPPLEMENTARY MATERIAL}

The supplementary material is available in the electronic version of this article: https://dx.doi.org/ 10.3233/BLC-190252.

\section{REFERENCES}

[1] Vaughn DJ, Broome CM, Hussain M, Gutheil JC, Markowitz AB.Phase II trial of weekly paclitaxel in patients with previously treated advanced urothelial cancer. J Clin Oncol. 2002;20:937-40.

[2] McCaffrey JA, Hilton S, Mazumdar M, Sadan S, Kelly WK, et al. Phase II trial of docetaxel in patients with advanced or metastatic transitional-cell carcinoma. J Clin Oncol. 1997;15:1853-57.

[3] Bellmunt J, Théodore C, Demkov T, Komyakov B, Sengelov $\mathrm{L}$, et al. Phase III trial of vinflunine plus best supportive care compared with best supportive care alone after a platinum-containing regimen in patients with advanced transitional cell carcinoma of the urothelial tract. J Clin Oncol. 2009;27:4454-61.

[4] Bellmunt J, De Wit R, Vaughn DJ, Fradet Y, Lee JL, et al. Pembrolizumab as second-line therapy for advanced urothelial carcinoma. N Engl J Med. 2017;376:1015-26.

[5] Rosenberg JE, Hoffman-Censits J, Powles T, Van Der Heijden MS, Balar AV, et al. Atezolizumab in patients with locally advanced and metastatic urothelial carcinoma who have progressed following treatment with platinum-based chemotherapy: a single-arm, multicentre, phase 2 trial. Lancet. 2016;387:1909-20.

[6] Sharma P, Retz M, Siefker-Radtke A, Baron A, Necchi A, et al. Nivolumab in metastatic urothelial carcinoma after 
platinum therapy (CheckMate 275): a multicentre, singlearm, phase 2 trial. Lancet Oncol. 2017;18:312-22.

[7] Apolo AB, Infante JR, Balmanoukian A, Patel MR, Wang $\mathrm{D}$, et al. Avelumab, an anti-programmed death-ligand 1 antibody, in patients with refractory metastatic urothelial carcinoma: results from a multicenter, phase Ib study. J Clin Oncol. 2017;35:2117-24.

[8] Massard C, Gordon MS, Sharma S, Rafii S, Wainberg ZA, et al. Safety and efficacy of durvalumab (MEDI4736), an anti-programmed cell death ligand-1 immune checkpoint inhibitor, in patients with advanced urothelial bladder cancer. J Clin Oncol. 2016;34:3119-25.

[9] Petrylak DP, Sternberg CN, Drakaki A, de Wit R, Nishiyama $\mathrm{H}$, et al. RANGE, a phase III, randomized, placebocontrolled, double-blind trial of ramucirumab (RAM) and docetaxel (DOC) in platinum-refractory urothelial carcinoma (UC): Overall survival results [abstract]. Ann Oncol 2018;29(Suppl 8):mdy283-074.

[10] Petrylak DP, De Wit R, Chi KN, Drakaki A, Sternberg $\mathrm{CN}$, et al. Ramucirumab plus docetaxel versus placebo plus docetaxel in patients with locally advanced or metastatic urothelial carcinoma after platinum-based therapy (RANGE): a randomised, double-blind, phase 3 trial. Lancet. 2017;390:2266-77.

[11] Eisenhauer EA, Therasse P, Bogaerts J, Schwartz LH, Sargent D, et al. (2009) New response evaluation criteria in solid tumours: revised RECIST guideline (version 1.1). Eur J Cancer 45:228-247.

[12] U.S. Department of Health and Human Services, National Institutes of Health, National Cancer Institute. Common Terminology Criteria for Adverse Events (CTCAE), v4.0 (v4.03). https://www.eortc.be/services/doc/ctc/CTCAE_ 4.03_2010-06-14_QuickReference_5x7.pdf. Updated June 14, 2010. Accessed January 09, 2019.
[13] Kaplan EL, Meier P. Nonparametric estimation from incomplete observations. J Am Stat Assoc. 1958;53:457-81.

[14] Cox DR. Regression models and life-tables. J R Stat Soc Series B Methodol 1972;34:187-220.

[15] Bellmunt J, Orsola A, Leow J, Wiegel T, De Santis M, et al. Bladder cancer: ESMO Practice Guidelines for diagnosis, treatment and follow-up. Ann Oncol 2014;25(Suppl 3): iii40-48.

[16] El Rassy E, Assi T, Bakouny Z, Pavlidis N, Kattan J. Beyond first-line systemic treatment for metastatic urothelial carcinoma of the bladder. Clin Transl Oncol 2019;21:280-88.

[17] Powles T, Durán I, van der Heijden MS, Loriot Y, Vogelzang $\mathrm{NJ}$, et al. Atezolizumab versus chemotherapy in patients with platinum-treated locally advanced or metastatic urothelial carcinoma (IMvigor211): a multicentre, open-label, phase 3 randomised controlled trial. Lancet. 2018;391: 748-57.

[18] Powles T, O’Donnell PH, Massard C, Arkenau HT, Friedlander TW, et al. Efficacy and safety of durvalumab in locally advanced or metastatic urothelial carcinoma: updated results from a phase 1/2 open-label study. JAMA Oncol 2017;3:e172411.

[19] Petrylak DP, Arkenau HT, Perez-Gracia JL, Krebs M, Santana-Davila R, et al. A multicohort phase I study of ramucirumab $(\mathrm{R})$ plus pembrolizumab $(\mathrm{P})$ : Interim safety and clinical activity in patients with urothelial carcinoma [abstract]. J Clin Oncol 2017;35(6 Suppl): 349.

[20] Rassy EE, Bakouny Z, Aoun F, Haddad FG, Sleilaty G, et al. A network meta-analysis of the $\mathrm{PD}(\mathrm{L})-1$ inhibitors in the salvage treatment of urothelial bladder cancer. Immunotherapy 2018;10:657-63. 